\title{
Research on the Construction of Cold Chain Logistics Intelligent System Based on 5G Ubiquitous Internet of Things
}

\author{
Qi Hao Han $\mathbb{D}^{1,2}$ \\ ${ }^{1}$ Beijing University of Posts and Telecommunications, School of Economics and Management, Beijing 100876, China \\ ${ }^{2}$ Henan University of Animal Husbandry and Economy, School of Economics and Trade, Zhengzhou, Henan 450044, China \\ Correspondence should be addressed to Qi Hao Han; 81473@hnuahe.edu.cn
}

Received 28 September 2021; Accepted 27 October 2021; Published 23 November 2021

Academic Editor: Guolong Shi

Copyright ( 2021 Qi Hao Han. This is an open access article distributed under the Creative Commons Attribution License, which permits unrestricted use, distribution, and reproduction in any medium, provided the original work is properly cited.

\begin{abstract}
In the research on the construction of cold chain logistics intelligent system based on $5 \mathrm{G}$ ubiquitous Internet of Things as an emerging technology, the Internet of Things is penetrating into the logistics field with its technical advantages and changing the original industry form. According to the development status of cold chain logistics, this paper constructs a set of cold chain logistics intelligent system by using RFID technology, sensing equipment, GPS system, fuzzy PID hierarchical control, and other Internet of Things technologies and analyzes the impact of the cost sharing ratio of Internet of Things on the income of wholesalers, retailers, and supply chain. The example analysis shows that when retailers share all the adoption costs of the Internet of Things, the benefit of the whole fresh agricultural product cold chain logistics is the largest. This study provides a scientific basis for logistics decision-making for wholesalers and retailers and has important application value for improving the logistics efficiency of the whole fresh agricultural product cold chain.
\end{abstract}

\section{Introduction}

With the development of society, it has been difficult for the $4 \mathrm{G}$ network to meet the needs of intelligence. The arrival of $5 \mathrm{G}$ communication technology provides the foundation for the interconnection of all things we have been looking forward to, provides a guarantee for the continuous construction of informatization, and has an important impact on our social economy and life. The interconnection of all things is a qualitative leap for the development of the logistics industry and creates new opportunities for the logistics industry [1]. Cold chain logistics is a systematic project based on refrigeration technology, which requires the logistics system to always be in a low-temperature environment in all links of production, storage, transportation, and sales, so as to ensure product quality and reduce product loss. Cold chain logistics is usually used in fruit and vegetable products, meat, flowers, medical supplies, special plasma, and other products that are not easy to store and have high value [2]. Because the products of cold chain logistics transportation are usually related to food safety and people's health, its development has been paid more and more attention by the state. Information construction is very important to the development of logistics industry. Under the integration environment of high and new technologies such as artificial intelligence, VR, AR, Internet, and Internet of Things, how to fully realize the construction of cold chain logistics by using $5 \mathrm{G}$ technology is the key to the information development of cold chain logistics intelligent system [3].

Under the new situation, while the cold chain logistics system has made progress, there are still many substantive problems [4]. First, the equipment is not perfect. Some cold chain equipment is still in an old state and has not been updated in time. It directly leads to the failure to provide temperature guarantee for the transported materials, increases the consumption, and thus increases the economic cost of investment in cold chain logistics. Secondly, the cold chain system has not been established, and there is no certain guarantee system for many kinds of food transportation, which lacks guidance and constraints on cold chain management. There is also an increase in food safety hazards, and the participation of third parties is not high [5]. The lack 
of guidance in cold chain management will lead to the poor control of food temperature in the process of transportation and eventually lead to the lack of guarantee of food quality, which can lead to the collapse of food transportation system. In addition, in the development of cold chain logistics, we pay more attention to urban food management and despise rural food management, which greatly affects the regional experience and benefits. The third party here mainly refers to outsourcing business, that is, some professional and technical personnel charge fees to implement outsourcing services for cold chain logistics. Their participation in the cold chain system is very low, which is not conducive to the technical and intelligent development of cold chain logistics. According to the forward-looking data survey, at present, about $40 \%$ of enterprises prefer self-supporting cold chain logistics business in order to effectively control product quality, but $60 \%$ still hope to reduce the pressure of product cold chain transportation with the help of thirdparty cold chain logistics. Cold chain logistics enterprises or enterprises that use the cold chain to transport goods throughout the process need corresponding standards to regulate their own cold chain transportation, so they should formulate cold chain operation standards through their own definitions, and also establish and improve their own brand effect. Typical enterprises include McDonald's, which adopts the international standard certification system. Refer to international standards to manage and control the whole process of cold chain transportation logistics [5]. If the Internet of Things technology is introduced into the cold chain logistics procurement, it can improve the procurement scheme and improve the overall work efficiency based on the effective application of RFID electronic tags and RFID readers. At present, RFID technology is more common in the identification and traceability of cold chain logistics, so as to improve the comprehensive level of food cold chain quality and safety. For the safety of food supply chain, at present, a few large international companies, especially cold chain enterprises, are using GPS/GPRS technology to track and supervise food transportation equipment from the perspective of logistics efficiency [6].

By building a networked intelligent warehousing information system model and cold chain logistics monitoring and tracking system, this paper faces the cold chain food and drug supply chain, so as to maintain quality, improve efficiency, reduce process cost, improve food safety supervision ability, and ensure that food adopts effective methods and tools while ensuring cold chain safety and transport in a suitable storage environment. Based on RFID technology, the ambient temperature of food storage in the supply chain is uploaded to the cloud to facilitate real-time monitoring of logistics data. The cold chain logistics system is responsible for analyzing the data collected from sensors and making decisions. Users obtain temperature data by interacting with the Internet of Things system.

\section{Related Work}

RFID system and sensor network have more advanced and mature applications in the United States, Britain, Germany,
Japan, Sweden, Switzerland, and South Africa. In the United States, Intel, Ti, and other American integrated circuit manufacturers have invested heavily in chip research and development [7]. Symbol and others have developed scanners that can read bar codes, and at the same time, IBM, Microsoft, and HP are also actively developing corresponding software and application systems to support the use of RFID and sensor networks [8]. In the United States, Internet of Things technology has been gradually applied in the fields of intelligent transportation, warehouse management and material tracking, production line automation and control, identity recognition, and so on. In the field of logistics, more than 100 enterprises in the United States have supported the application of RFID technology, including retailer giant Walmart; Large manufacturers Gillette, Johnson \& Johnson, and P \& G group; UPS, the world's largest express carrier and parcel delivery company; and the logistics application of the government in the Ministry of Defense [9]. In Japan, one or two RFID tags [10] are often placed in some trucks transporting valuable alcohol and food so as to monitor the temperature changes inside the carriage during delivery at any time to ensure its safety. In the medical and health field, RFID technology is mainly used to track medical waste with pollution hazards such as medical waste [11]. As a special cold chain product directly related to people's life safety, drugs have been deeply studied in the science, technology, and management of drug cold chain transportation in various countries. The supply chain system of refrigerated drugs in Japan has begun to take shape, and its management is very mature. Suppliers and distributors of upstream goods can share information, and RFID technology is widely used in the drug logistics industry [12], which makes the logistics center standardized and standardized. In addition, with highly automated storage and picking equipment, it is easy to operate and has powerful warehouse management. It can not only ensure the high efficiency of logistics operation of refrigerated drugs but also reduce the logistics cost. Food cold chain logistics is similar to the drug cold chain logistics process [13]; the cold chain logistics system based on Internet of Things technology has been basically completed in developed countries. The improvement of local technology and the application and improvement of new technology have become the focus of research on environmental protection, energy conservation, and safety. The infrastructure of food cold chain system is backward. At present, the proportion of perishable goods using cold chain logistics is small. In the market, many small and medium-sized enterprises do not have the ability to use refrigeration equipment because of their lack of funds and strength. Therefore, there is a lack of standardized fresh-keeping cold chain logistics cars and temperature control equipment. In order to save costs, many smaller companies cover the goods with cotton quilts to maintain the temperature during transportation, or the refrigeration equipment is closed after reaching a certain temperature, which will reduce the quality of perishable food.

The development of warehousing industry is becoming more and more automatic, intelligent, and informationbased [14]. Explore the further development of intelligent 
logistics warehousing informatization under 5G technology, design the intelligent logistics warehousing informatization system model under 5G technology according to the development difficulties of existing intelligent warehousing informatization, and analyze its operation process, characteristics, and functions [15]. At present, the operation mode of most intelligent storage information systems is that the terminal data at the operation layer is transmitted to the system layer through the data interface and then transmitted to the data cloud for processing, control, and storage, sending processing signals and reaching the operation layer through the system layer for operation instructions. The construction of intelligent warehousing information system model is an intelligent application based on $5 \mathrm{G}$ and other technologies. Its difference is that it can be decentralized [16]. During operation, the data at the end of the operation layer is directly uploaded to the data cloud for processing, and the system instructions and operation instructions are sent down after system processing.

\section{Analysis and Development of Cold Chain Logistics Intelligent System}

3.1. Design of Intelligent Logistics Warehousing Information System. The development of warehousing industry is becoming more and more automatic, intelligent, and informationbased. Explore the further development of intelligent logistics warehousing informatization under 5G technology, design the intelligent logistics warehousing informatization system model under 5G technology according to the development difficulties of existing intelligent warehousing informatization [17], and analyze its operation process, characteristics, and functions.

At present, the operation mode of most intelligent warehouse information systems is that the terminal data at the operation layer is transmitted to the system layer through the data interface and then transmitted to the data cloud for processing, control, and storage, sending processing signals and then reaching the operation layer through the system layer for operation instructions [18], as shown in Figure 1. The construction of intelligent warehousing information system model is an intelligent application based on $5 \mathrm{G}$ and other technologies. Its difference is that it can be decentralized. During operation, the data at the end of the operation layer is directly uploaded to the data cloud for processing, and the system instructions and operation instructions are sent down after system processing.

In the process of warehousing operation, goods are constantly warehoused. Therefore, it is necessary to count the inventory every other period of time and clean up some items with deteriorated or damaged quality. However, the traditional counting method has large time interval and large error between actual and system inventory information, resulting in the phenomenon of information asymmetry. After accessing 5G communication, the intelligent warehouse can more accurately conduct online real-time inventory on the system through the information sent by cargo pallets, so as to solve the problem of information asymmetry.
3.2. Overall Design of Intelligent Cold Chain Scheme. This paper comprehensively uses advanced sensing technology, wireless communication technology, monitoring and control technology, remote data transmission technology, satellite communication technology, and network and information technology to establish the whole process supervision system of cold chain logistics. The overall scheme is shown in Figure 2:

The basic information flow involved in this paper includes (1) offline link of food factory; (2) food storage; (3) food cold chain land transportation link; (4) food refrigerated transportation equipment parking link; (5) food cold chain and waterway transportation; and (6) the supervision link of food arriving on the other side. In order to support the system, realize the monitoring, tracking, and supervision of the whole food supply chain and ensure the safety of the food supply chain. To realize the real-time monitoring of food transportation environment, it is necessary to develop the cold chain intelligent monitoring terminal system. The intelligent monitoring terminal system includes an internal environment monitoring module and an intelligent terminal with GPS/GPRS/WiFi module to realize data acquisition and transmission.

The intelligent monitoring terminal system integrates a series of sensors such as temperature, humidity, and gas in the refrigerated container, which can monitor all conditions in the food transportation process and whether the food transportation process is safe, including whether the temperature and humidity are the required values for food storage. When fruits and vegetables and other foods are placed in the refrigerated container for transportation, the internal environment of the refrigerated container is monitored through advanced sensing and communication technology, including temperature, humidity, and internal gas concentration, so as to determine whether the internal environment of the refrigerated container is the temperature, humidity, and other environmental conditions required for food supply chain transportation, Monitor whether there are abnormal conditions causing deterioration or damage of food during transportation.

Through the cold chain intelligent monitoring terminal system developed in the project, information and data transmission is realized based on wireless communication modes such as GPRS, satellite communication, and WiFi and wired communication modes such as RS485/Ethernet. Based on database technology, an information platform for food cold chain (supply chain) safety supervision is developed to achieve the purpose of food packaging, storage, terminal sales, and transportation. Check the tracking and supervision of the whole supply chain. As a supervision realization, ensure that the food supply chain is transported with appropriate equipment and tools, and the food storage and transportation environment is not abnormal during the transportation of the food supply chain.

\subsection{Design of Real-Time Tracking Technology for Cold Chain} Logistics. This paper introduces the concept of intelligent cold chain. Based on the realization of food tracking and traceability, this paper realizes the real-time monitoring, 


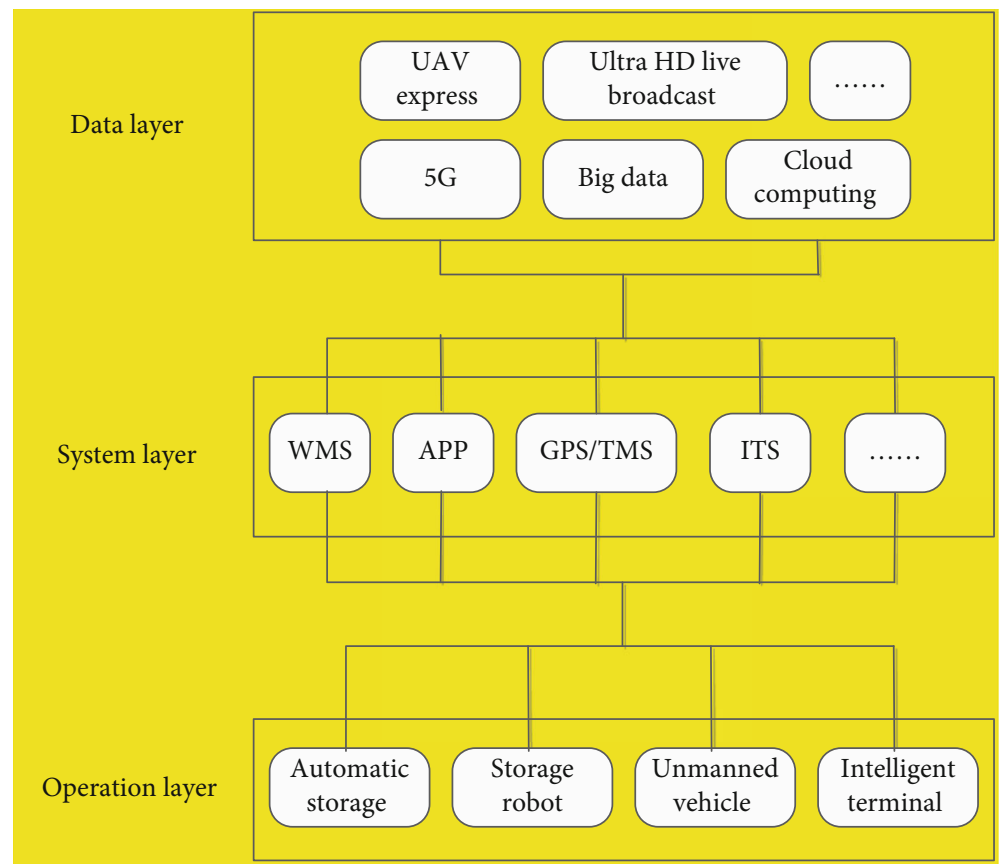

Figure 1: Model diagram of intelligent warehousing information system.

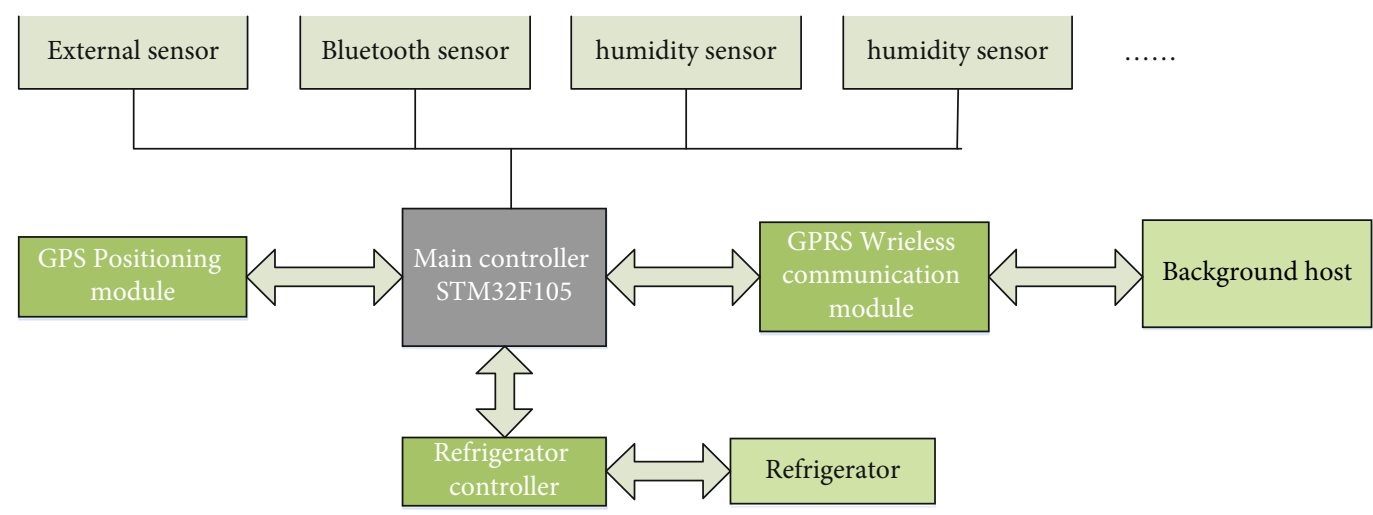

FIgURE 2: Structure framework of intelligent monitoring terminal system.

tracking, and supervision of the whole supply chain process from food packaging into finished products to storage, transportation, inspection, and terminal sales, so as to ensure the safety of food supply chain and provide effective technical means for food safety supervision for government regulators and serve business applications. The real-time tracking technology of cold chain logistics is as follows: develop an intelligent terminal integrating environmental temperature, humidity, and gas concentration monitoring and real-time monitoring of food storage environment with GPRS, WiFi wireless transmission, and RS485/Ethernet wired data transmission module [19]. The intelligent terminal for real-time monitoring of food storage environment can monitor the food storage environment in real time, including temperature, humidity, and gas concentration (such as oxygen, carbon dioxide, nitrogen, and ethylene), so as to identify the environmental conditions of food storage and whether there are abnormalities. Based on the application environment, the intelligent terminal for real-time monitoring of storage environment can transmit the food storage environment data to the monitoring center and background information system in real time through the wireless transmission mode of GPRS and WiFi and the wired data transmission mode of RS485/Ethernet, so as to realize the real-time monitoring of the environment in the case of food storage in the food supply chain. The food storage/container storage environment monitoring terminal is composed of environment detection and sensing, sensing wiring port, data processing control, information storage and analysis, expansion port, bus control, wired and wireless data transmission, power management, etc. Its structure is shown in Figure 3.

Figure 4 shows the design schematic diagram, and block 1 shows the module for wireless communication. In block 2, there is an intelligent monitoring terminal host, including wireless communication, GPS positioning and navigation, information storage and distribution, charging management, 


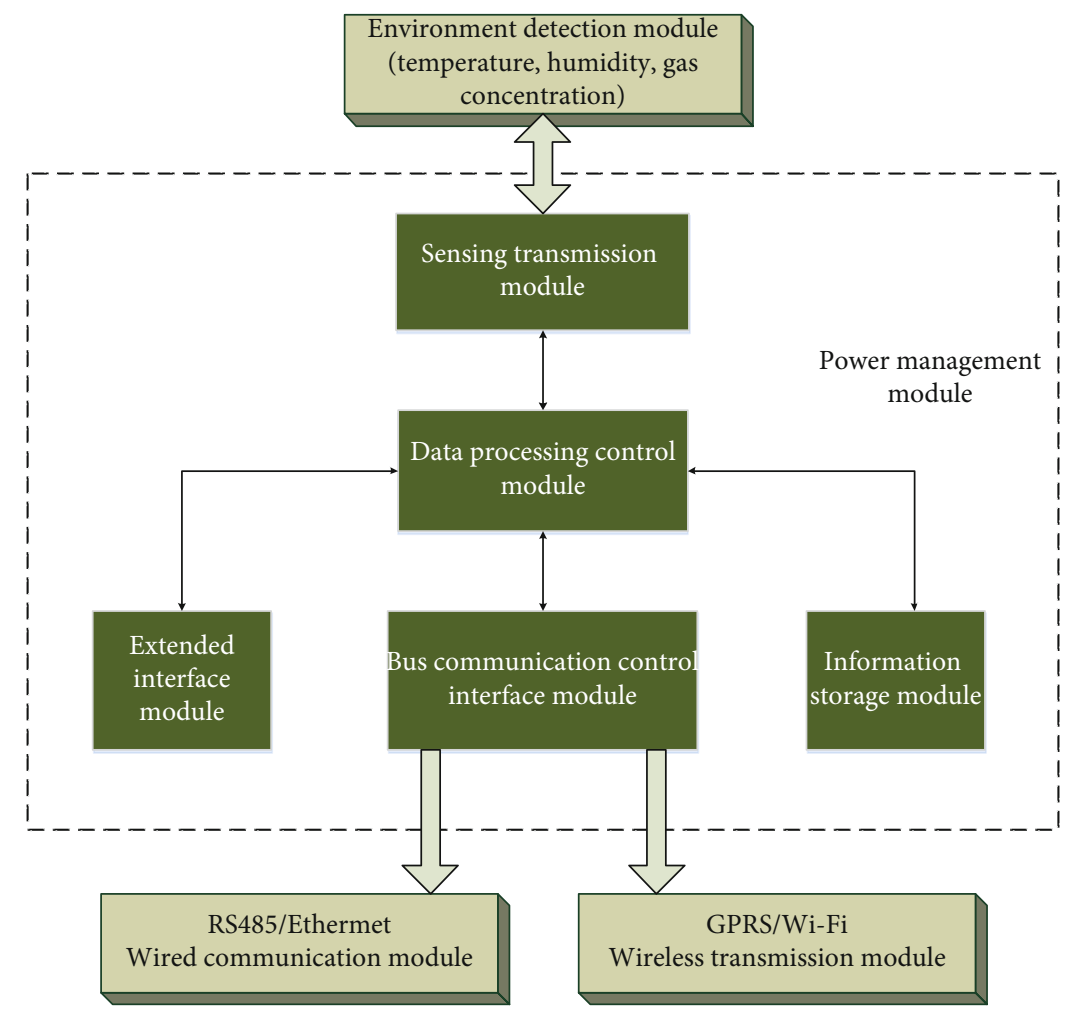

FIGURE 3: Module diagram of intelligent monitoring terminal for storage environment.

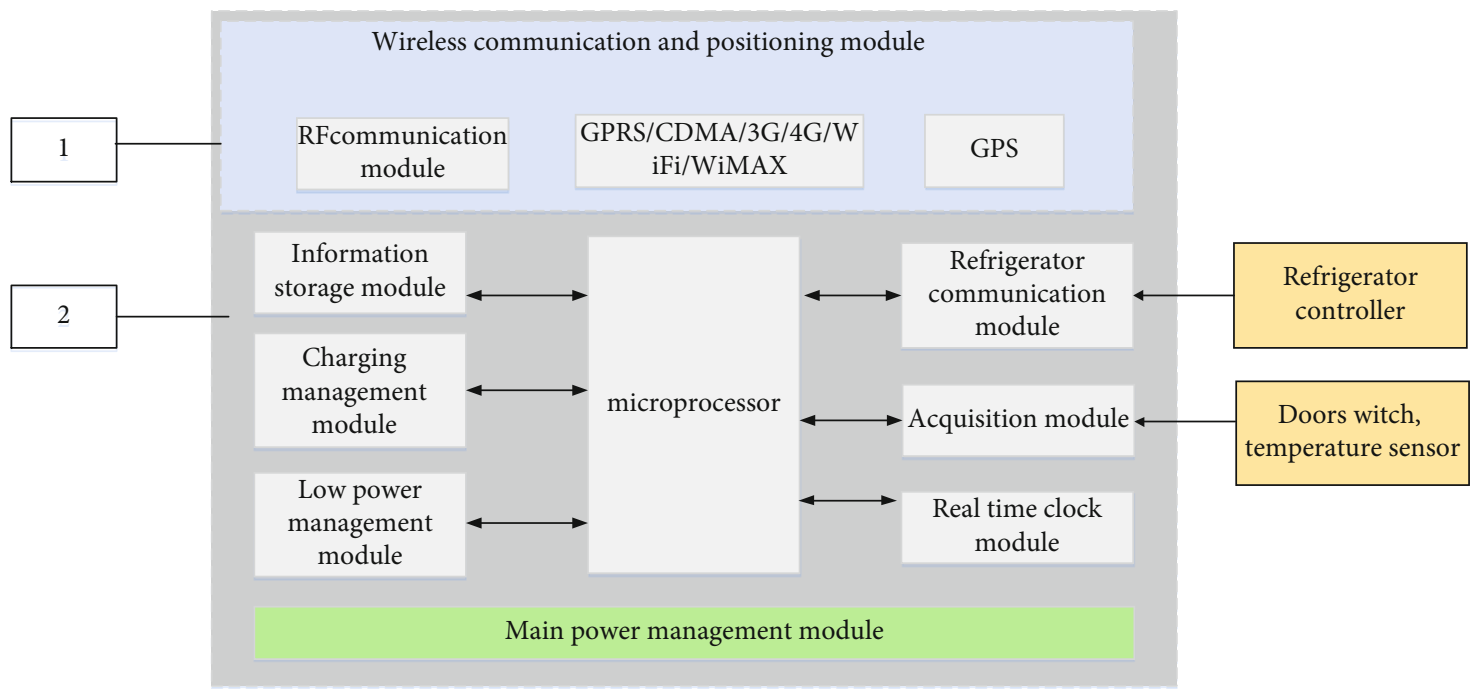

FIGURE 4: General composition diagram of intelligent monitoring terminal.

low-power processing, real-time clock, cold machine communication interface, acquisition and transmission, host power management, and other modules. The wireless communication module is composed of GPRS/CDMA/3G/4G/ WiFi/WiMAX wireless and satellite communication modules. The wireless communication module includes wireless tag, identification, contactless RF smart card, small wireless data terminal, safety and fire protection system, wireless remote control system, wireless 485/422 data communication, digital audio, digital image transmission, and other modules. At least one wireless communication function in the intelligent monitoring terminal host realizes data interactive communication with the background platform. The sensor unit and the refrigerator controller unit communicate with the intelligent monitoring terminal host.

3.4. Application Layer Overall Model Analysis. The object element of the application of Internet of Things technology in food cold chain logistics is all kinds of information in the circulation process of cold chain food [20], and its main body can be summarized into three parties: government regulators, consumers, and cold chain related enterprises. The 


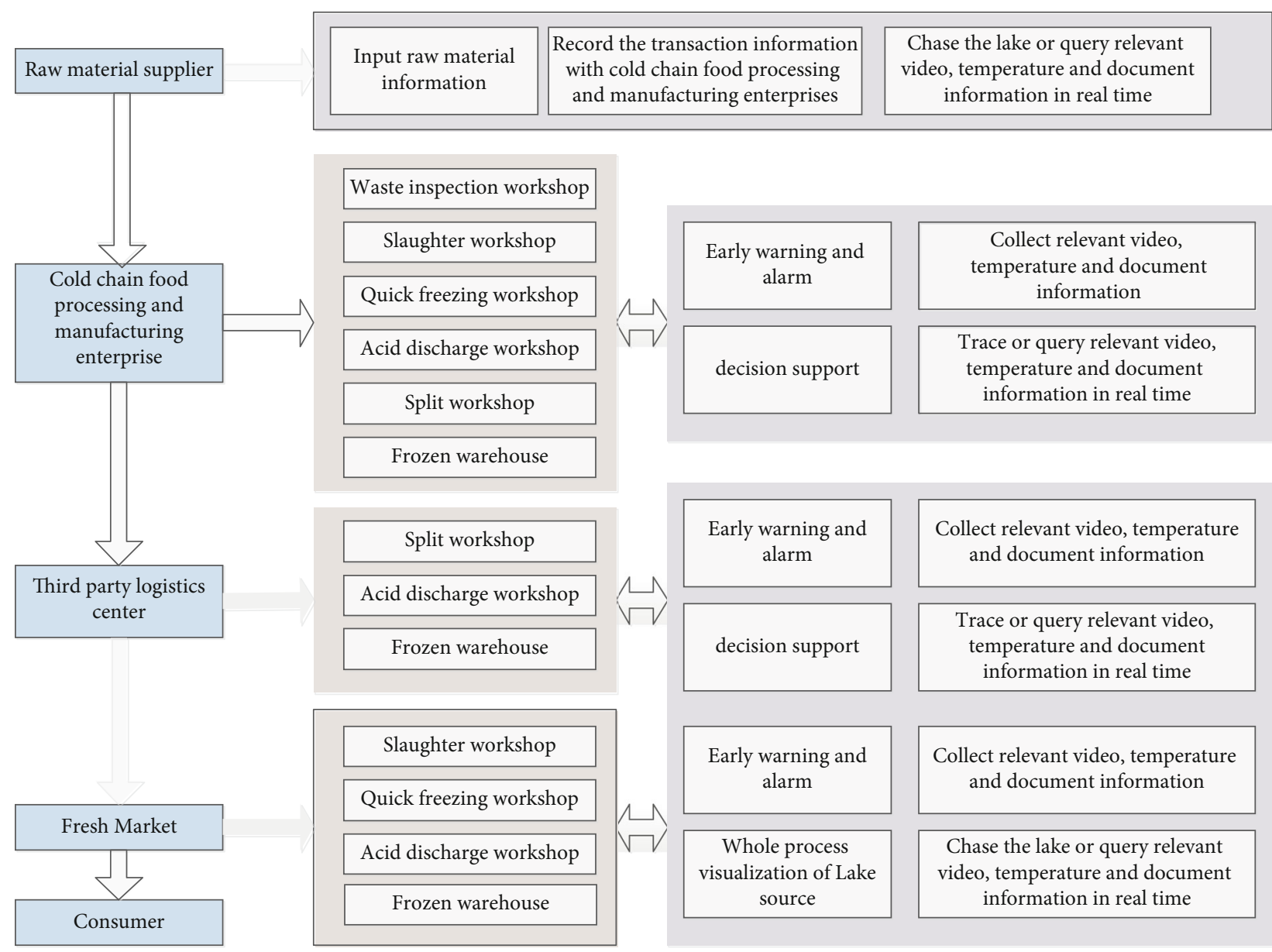

Figure 5: Overall functional requirements of the third party.

TABle 1: Data and equipment to be collected during transportation and distribution.

\begin{tabular}{lcc}
\hline Segment & Cold storage & Transport \\
\hline Collected data & Product information, cold storage temperature & Waybill information, GPS information, temperature, \\
Required sensors & Temperature sensor & Temperature sensor, door sensor \\
\hline
\end{tabular}

width, breadth, and depth of different subjects' demand for information are different. For the government regulatory department, it must have the ability to communicate effectively with cold chain-related enterprises; For cold chainrelated enterprises, in order to meet the needs of actual production and operation, they should master various data within the enterprise (of course, different organizational departments will have different permissions) and transfer and exchange various information between upstream and downstream enterprises. For consumers, their demand for information is to ensure food safety and food freshness. Therefore, the overall functional requirements from the three parties are shown in Figure 5:

At present, most domestic distribution centers use bar code scanning technology as the main means of goods management, information collection, and traceability. However, with the continuous improvement of information level, the disadvantages of bar code scanning technology are becoming more and more prominent, and this model is based on the application of emerging Internet of Things technologies such as Internet of Things. This stage can be divided into two links: refrigeration and transportation and distribution. In the refrigeration process, in addition to verifying the information in the previous stage, it is also necessary to integrate the information in this process, such as temperature information, necessary packing speed information, and contact personnel information. In particular, it is necessary to do a good job in the temperature control of the cold storage [21]. In the distribution and transportation link, the temperature and other information on the loading and unloading platform and transportation vehicles are the focus of monitoring at this stage. The data and equipment to be collected in this stage are shown in Table 1:

In the refrigeration process, the goods information includes goods code, storage mode, storage workshop, and management personnel information. It is necessary to 

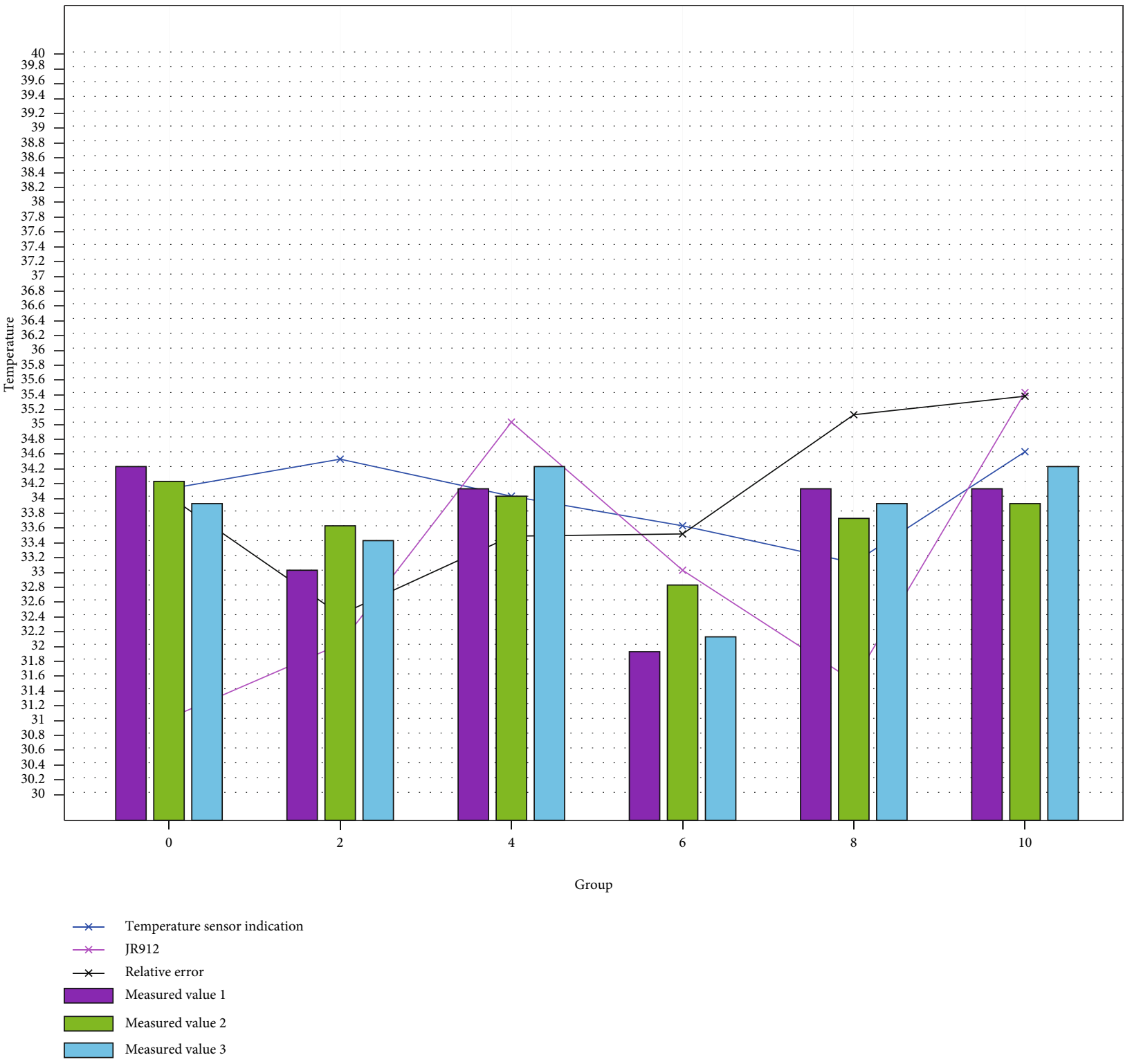

Figure 6: Multipoint temperature measurements and fusion values.

submit the product and product transportation status, distribution information, and distributor information during transportation. In the transportation and distribution link, the waybill information includes the name of the distributor, mode of transportation, means of transportation, sanitary environment, receiving and shipping place, date, quantity, and other information of the transported pork. The main carrier in the distribution process is the refrigerated vehicle, which is equipped with equipment. At the same time, the driver of each refrigerated vehicle has a card, and the driver can be associated with the refrigerated vehicle he drives through the reader. In the process of distribution, the system can locate the vehicle position in real time, and the relevant data can be transmitted to the background system through the network.

For the transportation and distribution stage of cold chain logistics, it is more important to monitor the temper- ature and door opening and closing information of refrigerated cars during transportation. The temperature sensor and door sensor installed in the refrigerator compartment obtain the temperature data and door opening and closing information in real time and then transmit them to the label connected to it for storage. When the tag receives the signal from the reader, it transmits the temperature data and door opening and closing data in the tag chip to the reader and then uses the technology to transmit the monitored data to the background system in real time. The real-time temperature change can be traced on the monitoring system. The corresponding early warning mechanism is set in the refrigerated vehicle and the background system. Once the temperature is too high or the door of the refrigerated compartment is abnormally opened, the alarm can be given at the first time to deal with the problem. 


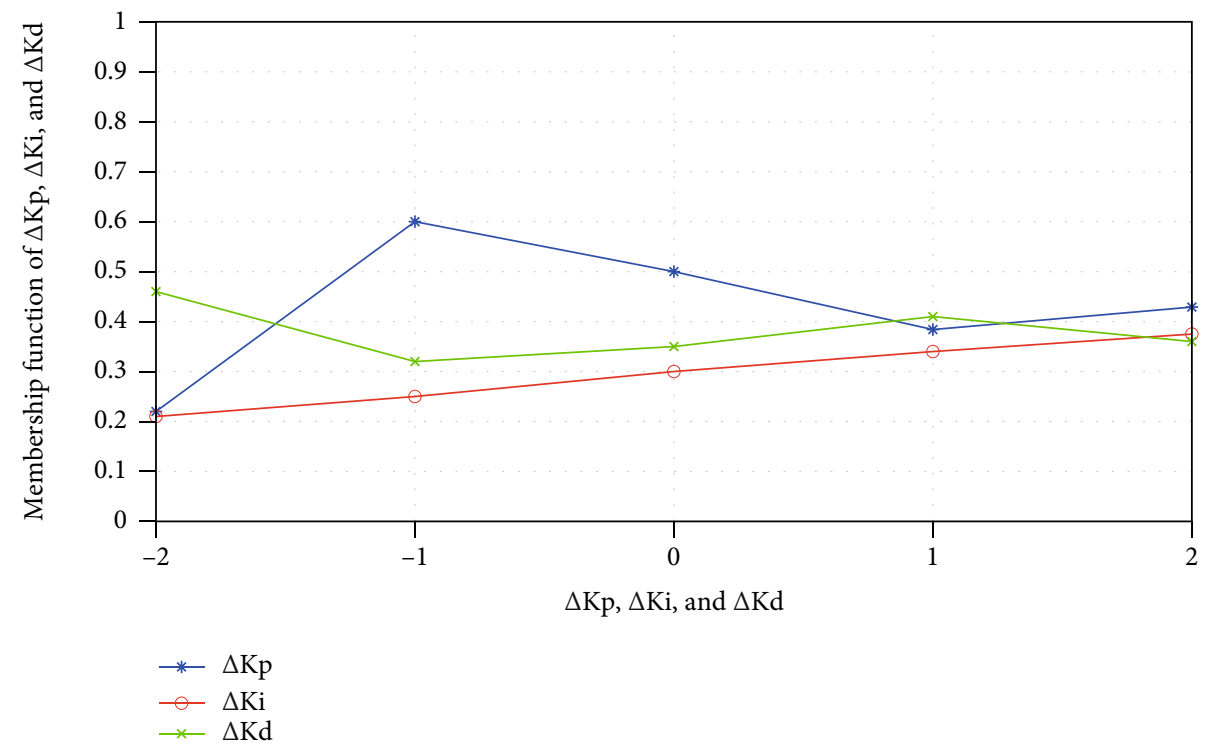

Figure 7: Membership functions of $\Delta \mathrm{Kp}, \Delta \mathrm{Ki}$, and $\Delta \mathrm{Kd}$.

\section{Experiment and Analysis}

4.1. System Implementation. The system uses Visual Studio 2008 integrated development tool and C\# advanced programming language under the framework of asp.net. The background database uses SQLServer2005 to store longitude, latitude, and temperature data. $\mathrm{C \#}$ is a programming language designed for the net development platform. C\# is closely combined with net. Various advantages of net are reflected through $\mathrm{C} \#$ as one of the net service providers; C\# uses the net class library. These libraries also support other development languages of the .Net platform. C\# is closely combined with web technology. C\# realizes the unification of application solution and web standard through soap (Simple Object Access Protocol). With the help of web service framework, programmers can use the existing object-oriented programming knowledge and skills to develop web services.

The user sets the cycle of uploading environmental information and the upper and lower limits of room temperature by the acquisition terminal, intelligently adjusts the environment in the greenhouse through Grubbs mean filtering and fuzzy PID hierarchical control algorithm, judges the system adjustment performance through data curve and report analysis, and makes relevant scientific research on the growth environment of crops using historical data. In a set of measurement data, if individual data deviates far from the average value, this data is called "suspicious value." If judged by statistical methods, such as the Grubbs method, the "suspicious value" can be eliminated from this group of measurement data without participating in the calculation of the average value. The temperature data of the same greenhouse are fused by Grubbs mean filtering algorithm, and the results shown in Figure 6 are obtained.

Data analysis is performed in group 1 and group 4, respectively: group 1 : $\mathrm{Ti}=34.46, \mathrm{Vi}=[-0.06,-0.06,0.14,-0.06$,
TABle 2: Fuzzy control rules for $\Delta \mathrm{Kp}$.

\begin{tabular}{|c|c|c|c|c|}
\hline \multirow{2}{*}{ Ec } & \multicolumn{4}{|c|}{$E$} \\
\hline & NB & NM & NS & $Z$ \\
\hline $\mathrm{NB}$ & $\mathrm{PB}$ & $\mathrm{PB}$ & $\mathrm{PM}$ & $\mathrm{PM}$ \\
\hline NM & $\mathrm{PB}$ & $\mathrm{PB}$ & $\mathrm{PM}$ & $\mathrm{PM}$ \\
\hline NS & $\mathrm{PM}$ & $\mathrm{PM}$ & $\mathrm{PM}$ & PS \\
\hline$Z$ & $\mathrm{PM}$ & $\mathrm{PM}$ & $Z$ & NS \\
\hline
\end{tabular}

$0.04], \sigma 1=0.08944, \mathrm{~g} 0 \times \sigma 1=0.14936$; group $4: \mathrm{Ti}=31.56$, $\mathrm{Vi}=[-0.46,-0.46,1.84,-0.46,-0.46], \sigma 1=1.02859$, and $\mathrm{g} 0$ $\times \sigma 1=1.71774$, where $\mathrm{Ti}$ is the arithmetic mean, $\mathrm{Vi}$ is the residual error, $0<i<6$, and $\sigma 1$ is the approximate error. By looking up the table, g0 $(5,0.05)=1.67$. There are no suspicious values in group 1 to be eliminated, and there are 33 in group 4.40 is a suspicious value, which needs to be eliminated. Calculate the mean value of the eliminated data, and the fusion values in group 1 and group 4 are 33.46 and 31.10, respectively. The relative difference between the fusion value and the temperature value measured by JR912 is $0-1.03 \%$, which meets the temperature accuracy requirements of cold chain transportation.

4.2. Fuzzy Design. If the model of a system can be easily obtained, the model can be analyzed and the controller suitable for the system model can be designed. But in reality, almost all systems are nonlinear and unpredictable. This requires us to fuzzy design the algorithm. The input and output of fuzzy reasoning are accurate quantities, but they are fuzzy quantities, which require the fuzzification, fuzzy decision-making, and defuzzification of accurate quantities in the process of algorithm implementation. Fuzzy design mainly includes two aspects: one is the number of fuzzy variables; the other is the design of fuzzy membership function. $\Delta \mathrm{Kp}$ is the system that selects $E$ and Ec as the input of fuzzy 


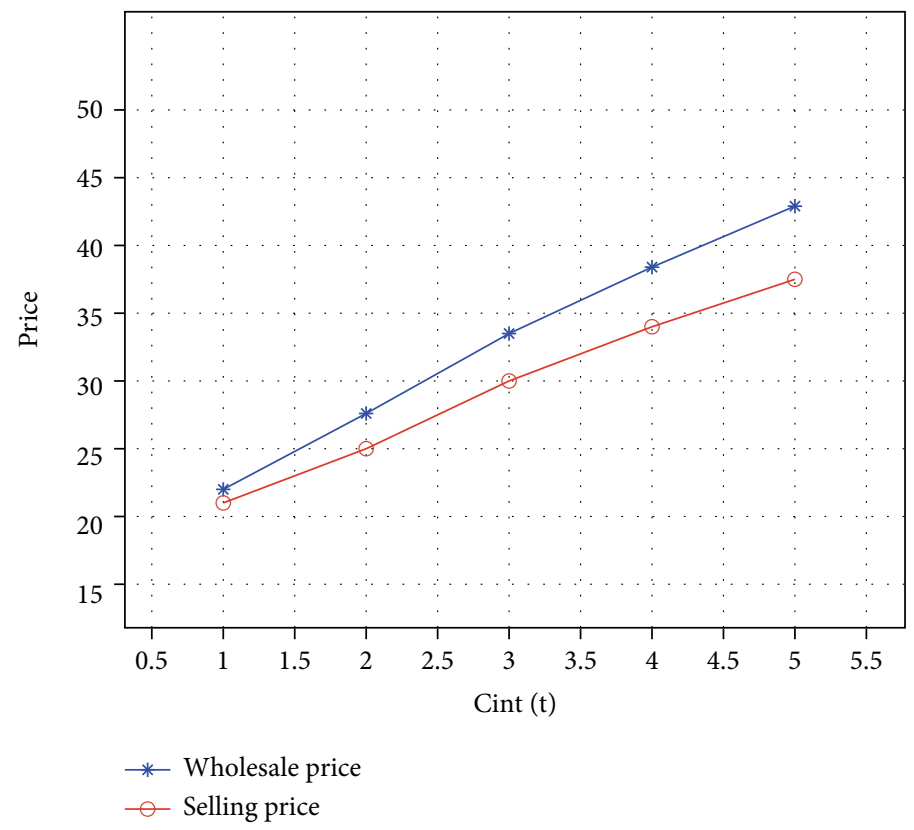

Figure 8: Broken line chart of the impact of $\operatorname{Cint}(t)$ on wholesale price and sales price.

controller; $\Delta \mathrm{Kp}, \Delta \mathrm{Ki}$, and $\Delta \mathrm{Kd}$ are the output variable of fuzzy controller, $E$, EC, $\Delta \mathrm{Kp}, \Delta \mathrm{Ki}$, and $\Delta \mathrm{Kd}$. The fuzzy subsets of $\Delta \mathrm{Kd}$ are defined as $\mathrm{Nb}$ (negative large), $\mathrm{nm}$ (negative medium), NS (negative small), $Z$ (zero), PS (positive small), $\mathrm{PM}$ (positive medium), and $\mathrm{Pb}$ (positive large), where the universe of $E$ and EC is set as $[-6,6]$ and for $\Delta \mathrm{Kp}, \Delta \mathrm{Ki}$, $\Delta \mathrm{Kd}$ is $[-0.3,0.3],[-0.06,0.06]$, and $[-3,3]$, respectively. The membership function setting of system parameters is shown in Figure 7.

Summarize the technical knowledge and actual operating experience of the engineering designer, as well as the interaction and influence of the $\Delta \mathrm{Kp}, \Delta \mathrm{Ki}$, and $\Delta \mathrm{Kd}$ parameters, and establish a fuzzy control rule table of $\Delta \mathrm{Kp}, \Delta \mathrm{Ki}$, and $\Delta \mathrm{Kd}$ accordingly. Take $\Delta \mathrm{Kp}$ as an example; as shown in Table 2, the system uses the average maximum membership method to solve the fuzzy. During the operation of the system, the control system completes the automatic correction of PID parameters and carries out hierarchical operation control through the analysis, table lookup, and calculation of fuzzy logic results. By introducing the binary control mechanism into the usual PID controller, the automatic correction of parameters can be realized.

4.3. Analysis of the Cost of the Internet of Things Technology on the Cold Chain Logistics System. Fresh agricultural products have the characteristics of easy loss. Scholars pay more and more attention to how to improve the efficiency of cold chain logistics of fresh agricultural products. The adoption of Internet of Things technology in cold chain logistics of fresh agricultural products can realize location tracking and information operation in transportation, storage, circulation, and processing. Taking the two-level cold chain logistics of one-way supply of fresh agricultural products (composed of wholesalers and retailers) as the research object, this paper studies the impact of the cost of adopting
Internet of Things technology and the proportion of each circulation subject sharing the cost of adopting Internet of Things technology on the relevant logistics decisions of the cold chain logistics. For wholesalers, transportation cost, warehouse cost, product cost, product loss, and shared IOT technology cost will affect their income. For retailers, the shared Internet of Things technology cost, product wholesale price, retail price, and order quantity affect their income. Due to the changes in the quality of agricultural products caused by the adoption of Internet of Things technology, the introduction of discount strategy to control retail prices and the construction of flexible and accurate replenishment and ordering models also belong to the cost of Internet of Things.

Assuming that the unit cost of adopting the Internet of Things technology is Cint $(t)$, the wholesaler's share of the cost of adopting the Internet of Things is $r$, and the retailer's share of the cost is $1-r$. The value of $r$ affects product transportation costs, warehouse costs, product wholesale prices, retail prices, order quantities, and the benefits of wholesalers, retailers, and the entire cold chain logistics.

Let $r=0.5$; $\operatorname{Cint}(t)$ takes the value of $0,0.5,1.0,1.5,2.0$, 2.5 , and 3.0, respectively, to analyze and adopt the unit cost of the Internet of Things to the wholesale price, retail price, order quantity, wholesalers, and retailers and the impact of the unit product revenue of the secondary cold chain. It can be seen from Figure 8 that wholesale prices and retail prices increase as the unit cost of adopting IoT technology increases. Retailers react more sensitively and have a larger growth rate. For wholesalers and retailers, with the increase of $\operatorname{Cint}(t)$, the unit product cost is greater than the unit revenue, and the order quantity presents a decreasing trend of quadratic concave function. The mathematical relationship between the retailer's order quantity and $\operatorname{Cint}(t)$ is shown in the following formula: 


$$
Q=\beta(t)\left[M^{w} \varphi^{-1}(t)\right]^{-k}\left(1-k^{-1}\right)^{2 k}
$$

With the increase of $\operatorname{Cint}(t)$, the unit product revenues of wholesalers, retailers, and the secondary cold chain are all showing an increasing trend.

\section{Conclusion}

It is of great significance to establish and improve the customer's cold chain transportation management system to view the internal state of the refrigerator and the operation data of the cold box in real time, so as to meet the requirements of customers for a comprehensive and detailed understanding of the operation of the cold box and to diagnose various faults of the refrigerator remotely. Taking the twolevel cold chain logistics of one-way supply of fresh agricultural products (composed of wholesalers and retailers) as the research object, this paper studies the impact of the cost of adopting Internet of Things technology and the proportion of each circulation subject sharing the cost of adopting Internet of Things technology on the relevant logistics decisions of the cold chain logistics. For wholesalers, transportation cost, warehouse cost, product cost, product loss, and shared IoT technology cost will affect their income. For retailers, the shared Internet of Things technology cost, product wholesale price, retail price, and order quantity affect their income. To sum up, the application of the Internet of Things in the cold chain logistics system can not only control the cost expenditure but also improve the practical work efficiency. Therefore, in order to better respond to the increasingly demanding market environment, cold chain logistics enterprises should increase their understanding and understanding of the Internet of Things technology and put forward new application strategies in combination with their own development needs.

\section{Data Availability}

The data used to support the findings of this study are available from the corresponding author upon request.

\section{Conflicts of Interest}

The author declares that there are no known competing financial interests or personal relationships that could have appeared to influence the work reported in this paper.

\section{References}

[1] K. Shafique, B. A. Khawaja, F. Sabir, S. Qazi, and M. Mustaqim, "Internet of things (IoT) for next-generation smart systems: a review of current challenges, future trends and prospects for emerging 5G-IoT scenarios," Ieee Access, vol. 8, pp. 2302223040, 2020.

[2] K. Sekaran, M. N. Meqdad, P. Kumar, S. Rajan, and S. Kadry, "Smart agriculture management system using internet of things," Telkomnika, vol. 18, no. 3, pp. 1275-1284, 2020.

[3] G. Tucker, "Sustainable product lifecycle management, industrial big data, and Internet of Things sensing networks in cyber-physical system-based smart factories," Journal of SelfGovernance and Management Economics, vol. 9, no. 1, pp. 919, 2021.

[4] V. Stehel, C. Bradley, P. Suler, and S. Bilan, "Cyber-physical system-based real-time monitoring, industrial big data analytics, and smart factory performance in sustainable manufacturing Internet of Things," Economics, Management, and Financial Markets, vol. 16, no. 1, pp. 42-51, 2021.

[5] S. Sicari, A. Rizzardi, and A. Coen-Porisini, " $5 \mathrm{G}$ in the internet of things era: an overview on security and privacy challenges," Computer Networks, vol. 179, p. 107345, 2020.

[6] A. Rejeb, J. G. Keogh, and H. Treiblmaier, "Leveraging the internet of things and blockchain technology in supply chain management," Future Internet, vol. 11, no. 7, p. 161, 2019.

[7] S. Kumar, P. Tiwari, and M. Zymbler, "Internet of Things is a revolutionary approach for future technology enhancement: a review," Journal of Big data, vol. 6, no. 1, pp. 1-21, 2019.

[8] X. Shi, X. An, Q. Zhao et al., "State-of-the-art internet of things in protected agriculture," Sensors, vol. 19, no. 8, p. 1833, 2019.

[9] X. Kong, F. Sun, X. Huo, X. Li, and Y. Shen, "Hierarchical optimal scheduling method of heat-electricity integrated energy system based on Power Internet of Things," Energy, vol. 210, p. 118590, 2020.

[10] S. Wijethilaka and M. Liyanage, "Survey on network slicing for Internet of Things realization in $5 \mathrm{G}$ networks," IEEE Communications Surveys \& Tutorials, vol. 23, no. 2, pp. 957-994, 2021.

[11] L. Siwu, X. Linzhi, M. Xiaodi, Z. Xiaobing, Y. Yulin, and P. Chan, "Integrated energy service platform under the umbrella of ubiquitous power internet of things," in Journal of physics: conference series, vol. 1346, no. 1, 2019IOP Publishing, 2019.

[12] R. Lowe, "Networked and integrated sustainable urban technologies in internet of things-enabled smart city governance," Geopolitics, History, and International Relations, vol. 13, no. 1, pp. 75-85, 2021.

[13] S. H. Alsamhi, O. Ma, M. S. Ansari, and F. A. Almalki, "Survey on collaborative smart drones and internet of things for improving smartness of smart cities," Ieee Access, vol. 7, pp. 128125-128152, 2019.

[14] I. Mistry, S. Tanwar, S. Tyagi, and N. Kumar, "Blockchain for 5G-enabled IoT for industrial automation: a systematic review, solutions, and challenges," Mechanical Systems and Signal Processing, vol. 135, p. 106382, 2020.

[15] M. A. Albreem, A. M. Sheikh, M. H. Alsharif, M. Jusoh, and M. N. M. Yasin, "Green Internet of Things (GIoT): applications, practices, awareness, and challenges," IEEE Access, vol. 9, pp. 38833-38858, 2021.

[16] E. Bourke, M. Kovacova, J. Kliestikova, and Z. Rowland, "Smart industrial internet of things devices, services, and applications: ubiquitous sensing and sensory data, predictive analytics algorithms, and cognitive computing technologies," Analysis and Metaphysics, vol. 18, pp. 50-56, 2019.

[17] M. Li, H. Liu, and X. J. Zhang, "Research on optimization model of location and layout of multi logistics distribution center," Journal of Chongqing Jiaotong University, vol. 36, no. 1, pp. 97-102, 2017.

[18] S. Sahmim and H. Gharsellaoui, "Privacy and security in internet-based computing: cloud computing, internet of things, cloud of things: a review," Procedia computer science, vol. 112, pp. 1516-1522, 2017. 
[19] T. Park, M. Zhang, and Y. Lee, "When mixed reality meets Internet of Things," GetMobile: Mobile Computing and Communications, vol. 22, no. 1, pp. 10-14, 2018.

[20] B. Mittelstadt, "Designing the health-related internet of things: ethical principles and guidelines," Information, vol. 8, no. 3, p. 77, 2017.

[21] I. F. Akyildiz and A. Kak, "The Internet of Space Things/CubeSats: a ubiquitous cyber-physical system for the connected world," Computer Networks, vol. 150, pp. 134-149, 2019. 